\title{
Validating a human model for anxiety using startle potentiated by cue and context: the effects of alprazolam, pregabalin, and diphenhydramine
}

\author{
J. M. P. Baas • N. Mol • J. L. Kenemans • \\ E. P. Prinssen • I. Niklson • C. Xia-Chen • F. Broeyer • \\ J. van Gerven
}

Received: 4 September 2008 / Accepted: 11 March 2009 /Published online: 5 May 2009

(C) The Author(s) 2009. This article is published with open access at Springerlink.com

\begin{abstract}
Background Fear-potentiated startle has been suggested as a translational model for evaluating efficacy of anxiolytic compounds in humans. Several known anxiolytic compounds have been tested as well as several putative anxiolytics. Because results of these studies have been equivocal, the aim of the present study was to examine another pharmacological permutation of the human potentiated startle model by comparing two anxiolytic agents to a non-anxiolytic sedative and placebo.

Methods Twenty healthy volunteers participated in a doubleblind, placebo-controlled, cross-over study with four sessions in which they received single doses of the anxiolytics alprazolam $(1 \mathrm{mg})$ and pregabalin $(200 \mathrm{mg})$, as well as diphenhydramine $(50 \mathrm{mg}$ ) as a non-anxiolytic sedative control and placebo. The design included a cued shock condition that presumably evokes fear and an unpredictable shock context condition presumably evoking anxiety.
\end{abstract}

J. M. P. Baas $(\bowtie) \cdot$ N. Mol $\cdot$ J. L. Kenemans

Department of Experimental Psychology,

Faculty of Social Sciences, Utrecht University,

Van Unnik Building, Heidelberglaan 2,

3584 CS Utrecht, The Netherlands

e-mail: j.m.p.baas@uu.nl

E. P. Prinssen • I. Niklson

CNS Research, F. Hoffmann-La Roche, Clinical Development,

Basel, Switzerland

C. Xia-Chen $\cdot$ F. Broeyer $\cdot J$. van Gerven

Centre for Human Drug Research,

Leiden, The Netherlands

Present address:

I. Niklson

Swissmedic,

Bern, Switzerland
Results None of the treatments reliably reduced either fearor anxiety-potentiated startle. Alprazolam and diphenhydramine reduced overall baseline startle. Alprazolam was found to only affect contextual anxiety in a statistical significant way after two subjects who failed to show a contextual anxiety effect in the placebo condition were excluded from the analysis. Pregabalin did not significantly affect any of the physiological measures.

Discussion The negative findings from this study are discussed in terms of methodological differences between designs and in variability of startle both between and within study participants.

Conclusion Even though fear-potentiated startle may be used to translate preclinical evidence to human populations, methodological issues still hamper the application of this model to early screening of putative anxiolytic drugs.

Keywords Fear - Anxiety · Fear-potentiated startle . Human model $\cdot$ Alprazolam $\cdot$ Pregabalin

\section{Introduction}

Fear-potentiated startle (FPS) has been suggested as a promising translational tool for screening putative anxiolytic substances (Grillon 2008). Nevertheless, pharmacological validation of this model has yielded mixed results. FPS was shown to be reduced with putative anxiolytic substances such as the glutamatergic substance LY354740 (Grillon et al. 2003) and testosterone (Hermans et al. 2006). An acute dose of citalopram exacerbated FPS, in line with initial anxiogenic effects observed in the clinic (Grillon et al. 2007). On the other hand, not all validation studies with a "golden standard" in anxiolytics, benzodiazepines, were 
successful. The first demonstration of the effects of a benzodiazepine on potentiated startle was in an emotion induction model using affective pictures (Patrick et al. 1996). However, in order to stay close to the original animal models (Davis et al. 1993), most attempts to validate human FPS with benzodiazepines have focused on versions of the fear-potentiated startle model that use threat of electric shocks (Grillon et al. 1993; Grillon et al. 1991).

Bitsios et al. (1999) were the first to validate a threat of shock FPS model with the benzodiazepine diazepam. In this model, threat of shock was alternated with intervals of safe and communicated by applying and removing the shock electrodes. Startle potentiation in the threat interval as compared to the safe intervals was significantly reduced by administration of $10 \mathrm{mg}$ but not $5 \mathrm{mg}$ of diazepam. This model has also been proven successful with lorazepam (2 mg, not $1 \mathrm{mg}$; Graham et al. 2005). In the model originally introduced by Grillon et al. (1993), Riba and colleagues reported no effect of the benzodiazepine lorazepam (4 mg, even though baseline startle was affected substantially; Riba et al. 1999) and a decrease in FPS after administration of $1 \mathrm{mg}$ of the benzodiazepine alprazolam (not 0.25 or $0.5 \mathrm{mg}$; Riba et al. 2001).

Earlier studies showed that the benzodiazepines oxazepam (15 and $30 \mathrm{mg}$ ) and diazepam (4, 10 and $15 \mathrm{mg}$ ) were not effective in reducing FPS in three different experiments (Baas et al. 2002a). Several factors were suggested to contribute to these conflicting results, the most important one being the exact kind of fear or anxiety responses evoked. Sensitivity of baseline startle in the context of a threat experiment to benzodiazepines suggested that perhaps contextual anxiety is sensitive to benzodiazepines, but cued fear is not (Baas et al. 2002a; Grillon 2002; Grillon and Baas 2003). Indeed, the manipulations validated by Bitsios et al. (1999) and Graham et al. (2005) and arguably also by Riba et al. (2001) have features of a context manipulation and thus may be assumed to cause a contextual anxiety response (Baas et al. 2002a; Grillon et al. 2006). On the other hand, results from a conditioning study showed that diazepam does not suppress the expression of a conditioned (i.e., cued) fear-potentiated startle response (Scaife et al. 2005). Therefore, even though not all permutations of the threat of shock FPS model were validated successfully, models that focus on contextual anxiety should lead to more consistent results (Grillon 2008).

In order to study both contextual anxiety and cued fear, a new threat of shock design was developed that contained both cued fear in a predictable shock condition and contextual anxiety evoked by an unpredictable shock condition (Grillon et al. 2004). This model was validated according to the hypotheses: While contextual anxiety was affected by $1 \mathrm{mg}$ alprazolam, cued fear was not (Grillon et al. 2006). This study also included diphenhydramine as non-anxiolytic sedative control and showed that this compound reduced baseline startle to the same extent as alprazolam $1 \mathrm{mg}$ while not having the specific effect on contextual fear. These results increased the evidence that especially contextual fear is influenced by benzodiazepine anxiolytics and that this effect cannot be solely attributed to sedation (Grillon and Baas 2003; Grillon and Baas 2002).

The present study aimed at extending the general validity of these results. The first aim was to replicate the dissociation between benzodiazepine effects on cued and contextual fear in another laboratory. The second aim was to extend the validation to an anxiolytic substance with another mechanism of action as benzodiazepines. The study included treatments with alprazolam and pregabalin as registered anxiolytics and diphenhydramine as a nonanxiolytic sedative control in 20 healthy volunteers. Pregabalin is registered as an anxiolytic substance for generalized anxiety disorder. Based on the hypothesized features that contextual anxiety and generalized anxiety share, a similar profile of efficacy as benzodiazepines was expected for pregabalin. Diphenhydramine was included as a non-anxiolytic sedative control. Doses were chosen based on a prior study in which alprazolam $1 \mathrm{mg}$ had significant effects in the FPS paradigm, and diphenhydramine $50 \mathrm{mg}$ produced sedative effects similar to alprazolam $1 \mathrm{mg}$ (Grillon et al. 2006). The pregabalin dose was chosen based on anxiolytic efficacy and similar expected levels of sedation.

\section{Methods}

Site

The study was implemented at the Centre for Human Drug Research in Leiden, the Netherlands.

Subjects

Subjects were recruited from the community by means of paper advertisements. Forty subjects provided informed consent and underwent a medical screening. Eight subjects were found not eligible for the study: Four subjects displayed reduced startle reactivity at screening, defined as no discernable response in at least three out of the 12 startle stimuli presented, and four subjects were excluded for medical reasons. Four subjects withdrew consent after screening. Twenty-four subjects participated (14 men, ten women) in the study, of whom 20 completed the study. Final sample characteristics were ten men and ten women, with a mean (SD) age of 22.4 (4.2) years and a Spielberger trait anxiety score of 31.8 (7.3). The study was performed according to ICH/GCP guidelines. The medical ethics committee of Leiden University Medical Center approved 
the study protocol, and all subjects gave written informed consent before participation.

\section{Treatments}

The study consisted of a double-blind full cross-over design with three active treatments (alprazolam $1 \mathrm{mg}$, pregabalin $200 \mathrm{mg}$, and diphenhydramine $50 \mathrm{mg}$ ) and placebo. Alprazolam is a benzodiazepine registered for the treatment of anxiety disorder. Pregabalin (Lyrica ${ }^{\circledR}$ ) is a relatively new compound registered for general anxiety disorder. It is supposed to modulate neuronal firing through binding to an auxiliary subunit $(\alpha 2-\delta)$ of voltage dependent calcium channels (Stahl 2004). Diphenhydramine is a H1antagonist with sedating but no documented primary anxiolytic properties upon acute treatment. All drugs were administered orally in identical capsules. Placebo consisted of an identical capsule containing no active ingredients. The active treatments alprazolam and pregabalin reach peak plasma concentrations around $1 \mathrm{~h}$ after dosing (Garzone and Kroboth 1989; Zareba 2005). Diphenhydramine reaches peak plasma concentration somewhat later at $2.3 \mathrm{~h}$ (Blyden et al. 1986), but sedative side effects as reflected in depressing effects on baseline startle are comparable to the effects of $1 \mathrm{mg}$ alprazolam when starting the FPS test $1 \mathrm{~h}$ after dosing (Grillon et al. 2006). Elimination half lives of alprazolam, pregabalin, and diphenhydramine are 9.5-12, 6, and $4 \mathrm{~h}$, respectively (Garzone and Kroboth 1989; Zareba 2005; Paton and Webster 1985). The order of the treatments of placebo (Pla), alprazolam (Alp), diphenhydramine (Dip), and pregabalin (Prg) was defined by a Williams Latin Square design (sequence 1: Pla, Prg, A1p, Dip; 2: Prg, Dip, Pla, A1p; 3: Alp, Pla, Dip, Prg; 4: Dip, A1p, Prg, Pla). Each subject was randomly assigned to one of the four sequences. Subjects who dropped out were replaced with another subject who received the same sequence. All four study days were identical and separated by washout periods of a week, with incidental deviations but guaranteeing a washout period of at least 3 days. After a standard breakfast $2 \mathrm{~h}$ before ingestion, baseline assessments were made, followed by administration of the study medication. The duration of the FPS paradigm was approximately $45 \mathrm{~min}$ and was performed between 1 and $2 \mathrm{~h}$ after dosing.

\section{Stimuli and apparatus}

Shock reinforcements were delivered through two disk electrodes located on the inside of the subjects' forearms using a Digitimer DS7A constant current stimulator (Digitimer, Hertfordshire, England). Stimulation consisted of short $(750 \mathrm{~ms})$ trains of 2-ms pulses delivered at $20 \mathrm{~Hz}$. A shock workup procedure was performed to individually set the level of shock used in the study. This workup consisted of three to five sample shocks, after each of which subjects rated how annoying/painful they found the preceding shock on a fivepoint scale. The level used in the study was the level at which a rating of 4 was achieved corresponding to "quite a bit painful/annoying". The startle reflex was evoked by bursts of white noise presented through headphones with $50-\mathrm{ms}$ duration and an intensity of $105 \mathrm{~dB}(\mathrm{~A})$. Task conditions as well as visual analog scales (VAS) were presented on a computer screen by automated scripts ran in the software package Presentation (Neurobehavioral Systems).

To assess eye blink startle response, EMG measurements were made from two electrodes on the lower orbicularis oculi muscle with a Grass Instruments system (Grass Technologies, West Warwick, USA). Skin conductance was recorded with a Grass Instruments skin conductance unit.

Subjects were asked to rate their level of emotional responding on a VAS at several times during the FPS procedure as an additional measure of emotional responding induced by the different task conditions. Included in these rating scales were questions about level of fear during all task conditions, i.e., during neutral, predictable, and unpredictable conditions, and in absence and presence of the visual cues. To assess levels of anxiety throughout the study day, subjects completed the state portion of the State-Trait Anxiety Inventory (STAI-DY1, Spielberger 1972) every hour. The trait anxiety levels reported on the screening day with the trait portion of the STAI are included in this paper.

\section{Experimental procedure}

The FPS task was included in a larger protocol assessing multiple dimensions of sedation as well as state anxiety and blood samples for pharmacokinetic/pharmacodynamic analysis at regular time intervals throughout the test day. This included several tests of central nervous system functioning (body sway, VAS scales of sedation (Bond and Lader 1974), adaptive tracking, saccadic eye movements, smooth pursuit eye movements). These data will be reported elsewhere. Main outcome parameter of the FPS task was startle magnitude across conditions, but skin conductance and subjective VAS ratings of fear and calmness were taken as additional measures of anxiety, in addition to a VAS rating of sedation (alertness).

The FPS procedure started with placement of electrodes on the orbicularis oculi muscle for startle measurement and on the palmar side of the left index and middle fingers for skin conductance recording. Each test session began with a series of nine startle stimuli presented with average intervals of $16 \mathrm{~s}$. After this, the shock electrodes were placed, and instructions concerning the FPS experiment 
were given. The FPS experiment contained three different contexts in which instructions regarding possible shock administration varied (Grillon et al. 2004, 2006). These contexts were signaled by a written text displayed on a computer monitor: "No shock" (Neutral context, N); "Shock only during cue" (Predictable context, P); and "Shock at any time" (Unpredictable context, U). In each context, cues were presented, e.g., red square for $\mathrm{N}$, blue circle for $\mathrm{P}$, and green triangle for $\mathrm{U}$ (assignment of stimuli to contexts counterbalanced between subjects). These cues were only predictive of a possible shock in the P context. Duration of contexts was 90-100 s, during which the verbal instructions concerning shock delivery remained on the screen and four cues were presented at regular time intervals with 8-s duration. Startle responding was assessed during all experimental conditions, i.e., during the presentation of these cues, as well as in their absence, which constituted the measure for contextual responding. Subjective ratings were also taken to differentiate between contexts and conditions of cue present and absent, but skin conductance responses are measured only in response to the discrete cues and to the onset of the context because a continuous measure of responding during the context cannot be derived.

The FPS test consisted of two blocks with the following predetermined orders of contexts: (1) P-N-U-N-U-N-P and (2) U-N-P-N-P-N-U. Each session consisted of both blocks, with the order of these two blocks counterbalanced across subjects. During each context, three startle probes were delivered in the absence and three in the presence of a cue, hence six per experimental condition per block and 12 in total across the experiment. Intervals between startle probes varied between 12 and $18 \mathrm{~s}$ (16 s on average). In each block, one of the two occurrences of the shock ( $\mathrm{P}$ and $\mathrm{U}$ ) contexts contained one shock reinforcement, and the other occurrence contained two shock reinforcements. Shock reinforcements during $\mathrm{P}$ contexts always coincided with a cue, hence predictably, while reinforcements during $U$ were administered in absence of the cue (statistically, some would coincide with a cue if administered randomly but these were excluded in order to prevent an association being made between the cue in the $\mathrm{U}$ context and the shocks). A total of 12 shocks were administered during each FPS test session. Orders of startle trials across experimental conditions and shock reinforcements were counterbalanced between blocks, sessions, and subjects. Each block began with three startle probes for habituation. After each block, subjective ratings on levels of fear specified for all experimental conditions were collected using computerized VAS scales [anchors $0=$ not fearful, $100=$ very fearful], as well as ratings on subjective state at that time, including fearfulness [same anchors], sedation [anchors $0=$ not alert, $100=$ very alert], and calmness [anchors $0=$ not at all calm, $100=$ very calm]. In addition, the subjective aversive- ness of shocks [anchors $0=$ not annoying, 100=very annoying] and startle probes [anchors $0=$ very uncomfortable, $100=$ very comfortable] was evaluated.

\section{Data analyses}

Analysis of the EMG signal was carried out in Brain Vision Analyzer software, and trials containing artifacts were removed according to established procedures (Blumenthal et al. 2005; Böcker et al. 2004; Mol et al. 2007). Startle magnitude was defined as the peak of the integrated signal in the period between 0 and $125 \mathrm{~ms}$ after presentation of the acoustic startle probe. For each participant, averages per condition across trials were calculated. There were two subjects who displayed relatively low startle magnitudes even after passing the baseline startle criterion at screening. Additional analyses were run excluding these subjects, which did not change any of the conclusions.

Skin conductance responses were quantified by scoring the peak response between 0.5 and $5 \mathrm{~s}$ after stimulus onset. Before statistical analysis, SCR data were normalized using a log transformation following Grillon et al. (2006).

All statistical analyses were repeated measures analyses of variance in SPSS 14 for Windows on averaged data per session and condition. Univariate (within-subjects) tests were reported, while Greenhouse Geisser epsilon corrections were applied for violations of the assumption of sphericity. In some cases, the multivariate tests yielded more power for the interactions with Drug, and in those cases, the multivariate tests are reported as well. Tests of planned primary contrasts using univariate contrasts for each drug condition against placebo were included and were reported in case these reached $(p<0.05)$ or approached $(<0.10)$ significance.

To test drug effects on FPS and VAS to explicit threat cues during the predictable shock condition, a Cue (Cue absent, Cue present) $\times$ Drug (4) ANOVA was employed. To test drug effects on FPS during the unpredictable shock context, a Context (Neutral context, Unpredictable context) $\times$ Drug (4) ANOVA was employed. An additional analysis on normalized data was included to assess the effects of reducing between-subjects variance caused by differences in baseline startle between subjects. Therefore, FPS analyses were repeated on $T$ scores calculated across all measurements per subject.

SCR are not evoked in absence of stimulation during the contexts; therefore, SCR to the fear cue in the predictable condition was analyzed with SCR to the cue in the neutral condition as a control, with factors Cue (Cue in neutral context, Cue in predictable shock context) $\times$ Drug (4). SCR increase to the onsets of the unpredictable context was analyzed with a Context (Neutral, Unpredictable) $\times$ Drug (4) ANOVA. 
Contribution of anxiolysis and sedation on drug effects on baseline startle (cf. Baas et al. 2002a) were assessed with correlation and regression analyses. For this purpose, average measures were taken across all conditions for both startle magnitude and the ratings of fearfulness during these conditions, and difference scores were taken based on the value during each of the drug conditions minus that value during the placebo condition. Along the same lines, alternative measures were derived for just the neutral conditions. The average score on the VAS alertness scale taken after each of the two blocks was employed as measure of sedation. In addition, to more generally explore other factors possibly contributing to differences in baseline startle, correlation analyses were done between these two measures of baseline startle (calculated based on all trials or based on just the trials during the neutral condition) and alertness and the two different measures of the VAS fearfulness ratings (calculated based on all conditions or based on just the neutral condition), plus the additional subjective ratings of subjective state right after each of the blocks (fearfulness and calmness, averaged across the measures per block) and two measures of the Spielberger State Anxiety score taken just prior and just after the FPS test. Of these, exploratory correlational analyses summaries were reported, and only significant correlations were highlighted.

\section{Results}

\section{FPS}

Startle magnitude was highly significantly increased in the predictable condition during the presence compared to the absence of the cue that predicted a shock (see Fig. 1, main

\section{Startle magnitudes in predictable context without / with cue:}

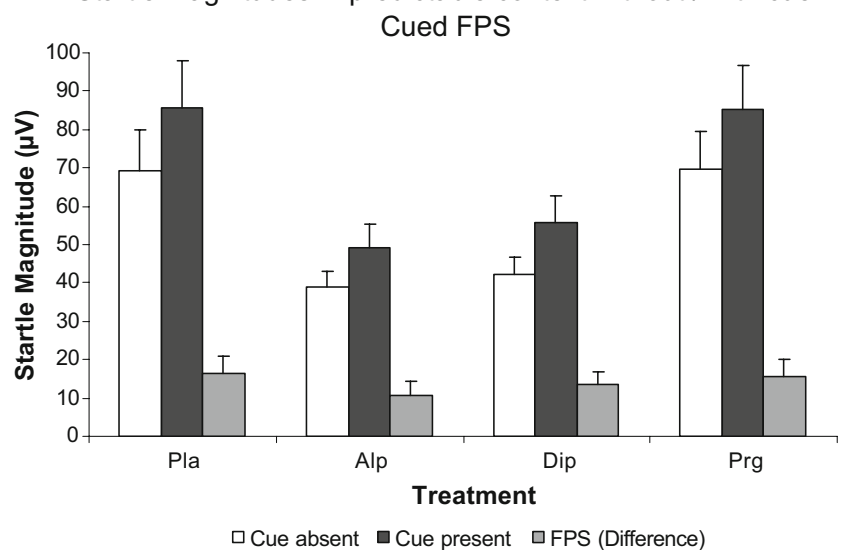

Fig. 1 Startle magnitudes in the predictable context reflecting cued FPS. Error bars are standard error of the mean. Pla placebo, Alp alprazolam, Dip diphenhydramine, Prg pregabalin
Startle magnitudes in predictable context without / with cue:

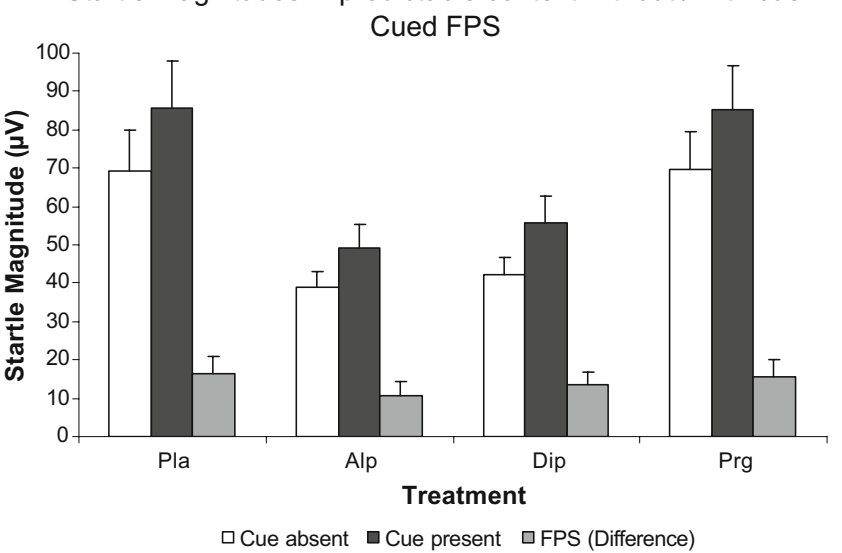

Fig. 2 Startle magnitudes in the unpredictable versus neutral contexts reflecting context FPS. Error bars are standard error of the mean. Pla placebo, Alp alprazolam, Dip diphenhydramine, Prg pregabalin

effect of Cue, $F(1,19 ; \mathrm{GG} \varepsilon=1)=24.4, p<0.001)$. Effects on overall startle magnitude were reflected in a main effect of Drug $(F(3,57 ; \mathrm{GG} \varepsilon=0.80)=7.4, p<0.001)$. Follow-up contrasts assessing differences from the placebo condition indicated significant reductions in overall startle magnitude by alprazolam $(F(1,19 ; \mathrm{GG} \varepsilon=1)=10.1, p<0.01)$ and diphenhydramine $(F(1,19 ; \mathrm{GG} \varepsilon=1)=9.6, p<0.01)$, but not pregabalin treatment $(F(1,19 ; \mathrm{GG} \varepsilon=1)=0.0$, NS). There was no significant effect of any of the active treatments on FPS (interaction Cue $\times$ Drug, $F(3,57 ; \mathrm{GG} \varepsilon=0.84)=0.5$, NS).

There was also a highly significant effect of the context manipulation on startle magnitudes (Fig. 2, main effect of Context $F(1,19 ; \mathrm{GG} \varepsilon=1)=24.3, p<0.001)$. Similar to the startle data from the specific cue condition, both alprazolam and diphenhydramine significantly reduced overall startle magnitude (main effect Drug $F(3,57 ; \mathrm{GG} \varepsilon=0.84)=8.0, p<$ 0.001 ; significant contrasts between alprazolam and placebo $F(1,19 ; \mathrm{GG} \varepsilon=1)=13.6, p<0.005$, diphenhydramine and placebo $F(1,19 ; \mathrm{GG} \varepsilon=1)=12.2, p<0.005$, pregabalin and placebo $F(1,19 ; \mathrm{GG} \varepsilon=1)=0.2$, NS). However, the expected interaction effect between context and treatment was not significant $(F(3,57 ; \mathrm{GG} \varepsilon=0.77)=1.4, \mathrm{NS})$.

In an additional post hoc analyses, two subjects were found to display very small potentiation (context U-context $N<$ $1.5 \mu \mathrm{V}$, which corresponds to $<3.1 \%$ of their startle level during context $\mathrm{N}$ ), and two subjects even showed the opposite of context potentiation of startle under placebo treatment (one subject scored $-10.0 \mu \mathrm{V}(-4.6 \%)$, the other $-11.2 \mu \mathrm{V}$ $(-5.3 \%))$. Analyses of the data excluding the latter two subjects $(N=18$, see hatched bars in Fig. 2) revealed that the Drug $\times$ Context interaction, including all four levels of the factor Drug did not reach significance in the univariate tests $(F(3,51)=1.7, p=0.19$, while a trend toward significance was observed in the multivariate test, $F(3,15)=3.1, p=0.06)$. Also, 
the specific contrast for placebo and alprazolam on the interaction of Drug $\times$ Context was significant $(F(1,17)=5.2$, $p<0.05)$, whereas the effects of diphenhydramine $(F(1,17)=$ $2.0, \mathrm{NS})$ and pregabalin $(F(1,17)=0.0, \mathrm{NS})$ were not.

Analyses on $T$ scores revealed highly significant effects of cued FPS $(F(1,19 ; \mathrm{GG} \varepsilon=1)=49.6, p<0.001)$ and context FPS $(F(1,19 ; \mathrm{GG} \varepsilon=1)=43.5, p<0.001)$ on startle magnitude, which were not affected by Drug (Cue $\times$ Drug $F(3,57$;GG $\varepsilon=0.84)=$ 0.4 , NS; Context $\times$ Drug $F(3,57 ; \mathrm{GG} \varepsilon=0.85)=0.1, \mathrm{NS})$.

VAS reports of subjective fear per condition

There was a very significant main effect of presence of the threat cue in the predictable condition on rating of fearfulness $(F(1,19 ; \mathrm{GG} \varepsilon=1)=27.9, p<0.001$, see Table 1$)$ and a main effect of drug on overall reported fearfulness during the predictable shock condition $(F(3,57 ; \mathrm{GG} \varepsilon=0.90)=4.9, p<$ $0.01)$. The effect of drug is due to reduced fearfulness in the alprazolam condition with respect to placebo $(F(1,19 ; \mathrm{GG} \varepsilon=$ $1)=10.7, p<0.005)$; the other treatments did not reach significance $(F$ values for diphenhydramine and pregabalin are 0.2 and 1.4 , respectively). The interaction between the effects of the fear induction and drug condition was not significant $(F(3,57 ; \mathrm{GG} \varepsilon=0.82)=1.4, \mathrm{NS})$.

The difference in VAS fearfulness data during the unpredictable and neutral contexts (Table 1) corresponds to contextual FPS. There was a significant main effect of threat context on rating of fearfulness $(F(1,19 ; \mathrm{GG} \varepsilon=1)=$ $57.1, p<0.001)$ and a main effect of drug on overall reported fearfulness during the unpredictable shock condition $(F(3,57 ; \mathrm{GG} \varepsilon=0.78)=3.5, p<0.05)$. The effect of drug was due to reduced fearfulness in the alprazolam condition (follow-up contrast comparing alprazolam to placebo: $F$ $(1,19 ; \mathrm{GG} \varepsilon=1)=5.8, p<0.05)$; the other treatments do not reach significance ( $F$ values for the comparisons of diphenhydramine and pregabalin to placebo are 0.1 and 1.6, respectively). The interaction between the threat manipulation and drug condition did not reach significance
$(F(3,57 ; \mathrm{GG} \varepsilon=0.96)=1.8, \mathrm{NS})$, although the condition effect tested specifically between placebo and alprazolam closely approached significance $(F(1,19 ; \mathrm{GG} \varepsilon=1)=4.4, p=$ $0.05, F$ values for diphenhydramine and pregabalin were, respectively, 2.8 and 0.2 , NS). The two subjects who displayed a negative context effect with startle also showed a small effect on their subjective ratings $(<3$ out of 100 points), but there were four other subjects with such a small difference in subjective fear reports between the neutral and unpredictable context. The exclusion of these two subjects yielded very similar results.

VAS reports on overall fear, alertness/calmness, and shock/ probe evaluation

Table 2 contains the VAS data on subjective ratings on fearfulness, alertness, calmness, and shock and probe evaluation taken after each of the two runs of the FPS procedure (averaged across runs). After runs of the FPS experiment, subjects reported feeling less anxious under pregabalin and less alert under alprazolam and diphenhydramine. All drug treatments increased subjective ratings of calmness slightly, but not significantly. Only alprazolam had a significant effect on evaluation of the shock.

Regression and correlation analysis of drug effects on baseline startle and subjective fear/sedation

The regression analysis indicated that differences in VAS fearfulness and sedation predicted drug-induced differences in baseline startle for none of the drugs (averaged across all conditions all values for $F(2,17)<1.3$, NS). When including only the neutral conditions, which may constitute a purer assessment of baseline state, the prediction for alprazolam approached significance $(F$ $(2,17)=3.1, p=0.07$; individual beta's: fearfulness beta= $0.57, t=2.5, p<0.05$, alertness beta $=-0.31, t=-1.3$, NS).

Table 1 VAS ratings of subjective fear per condition

\begin{tabular}{|c|c|c|c|c|c|c|c|c|c|c|c|c|}
\hline & \multicolumn{6}{|c|}{ Predictable context } & \multicolumn{6}{|c|}{ Unpredictable shock context versus neutral context } \\
\hline & \multicolumn{2}{|c|}{ Cue absent } & \multicolumn{2}{|c|}{ Cue present } & \multicolumn{2}{|c|}{$\begin{array}{l}\text { Cue fear potentiation } \\
\text { (difference) }\end{array}$} & \multicolumn{2}{|c|}{$\begin{array}{l}\text { Neutral } \\
\text { context }\end{array}$} & \multicolumn{2}{|c|}{$\begin{array}{l}\text { Unpredictable shock } \\
\text { context }\end{array}$} & \multicolumn{2}{|c|}{$\begin{array}{l}\text { Context fear potentiation } \\
\text { (difference) }\end{array}$} \\
\hline & Mean & SEM & Mean & SEM & Mean & SEM & Mean & SEM & Mean & SEM & Mean & SEM \\
\hline Pla & 32.45 & 4.99 & 56.75 & 5.94 & 24.30 & 4.92 & 14.38 & 3.73 & 54.63 & 5.36 & 40.25 & 5.49 \\
\hline Alp & 23.15 & 4.64 & 42.15 & 5.66 & 19.00 & 4.08 & 11.23 & 2.46 & 43.13 & 5.28 & 31.90 & 4.85 \\
\hline Dip & 32.15 & 5.69 & 53.95 & 5.96 & 21.80 & 4.95 & 19.23 & 3.48 & 52.35 & 5.70 & 33.13 & 5.05 \\
\hline Prg & 27.35 & 5.62 & 53.85 & 6.38 & 26.50 & 5.63 & 10.90 & 3.19 & 48.90 & 5.74 & 38.00 & 6.02 \\
\hline
\end{tabular}

SEM standard error of the mean, Pla placebo, Alp alprazolam, Dip diphenhydramine, Prg pregabalin 
Table 2 Mean (SEM) VAS rating of overall fearfulness, alertness, calmness, aversiveness of shock, and probe

\begin{tabular}{llcccc}
\hline & Placebo & Alprazolam & Diphenhydramine & Pregabalin & Main effect Drug \\
\hline Fearful & $34.2(4.2)$ & $29.9(3.9)$ & $26.3(3.9)$ & $24.1(4.2)$ & $F(3,57 ; \mathrm{GG} \varepsilon=0.77)=2.5, p=0.08^{\mathrm{a}}$ \\
Alert & $51.7(3.7)$ & $36.1(3.3)$ & $41.9(3.5)$ & $46.5(3.8)$ & $F(3,57 ; \mathrm{GG} \varepsilon=0.81)=6.4, p<0.005^{\mathrm{b}}$ \\
Calm & $56.9(4.1)$ & $64.9(4.0)$ & $64.6(3.8)$ & $65.1(4.0)$ & $F(3,57 ; \mathrm{GG} \varepsilon=0.78)=2.2, \mathrm{NS}$ \\
Shock & $52.7(3.8)$ & $41.0(5.4)$ & $50.6(4.3)$ & $46.0(4.6)$ & $F(3,57 ; \mathrm{GG} \varepsilon=0.83)=3.9, p<0.05^{\mathrm{c}}$ \\
Probe & $36.4(3.4)$ & $38.8(3.7)$ & $37.7(2.3)$ & $36.8(2.8)$ & $F(3,57 ; \mathrm{GG} \varepsilon=0.83)=0.2, \mathrm{NS}$ \\
\hline
\end{tabular}

SEM standard error of the mean, NS not significant

${ }^{a}$ Alp-Pla $F(1,19)=1.3$, NS; Dip-Pla $F(1,19)=2.8$, NS; Prg-Pla $F(1,19)=4.5, p<0.05$

${ }^{\mathrm{b}}$ Alp-Pla $F(1,19)=14.1, p<0.001$; Dip-Pla $F(1,19)=5.2, p<0.05$; Prg-Pla $F(1,19)=1.5$, NS

${ }^{\mathrm{c}}$ Alp-Pla $F(1,19)=7.0, p<0.05$; Dip-Pla $F(1,19)=0.3$, NS; Prg-Pla $F(1,19)=2.8$, NS

The same test for diphenhydramine and pregabalin remained nonsignificant (values for $F(2,17)<1.5$, NS).

As suggested by the marginal findings with the regression analyses, exploratory analyses found few correlations between subjective effects and effects of drugs on baseline startle. For alprazolam against placebo, none of the correlations reached statistical significance $(-0.21<$ all Pearson $r$ correlations $<0.44$, all $p$ values $>0.05)$. A marginally significant correlation was found between the VAS fear measures and baseline startle during the neutral condition (Pearson $r=0.44, p=0.05$ ). For diphenhydramine against placebo, there were also no significant correlations $(-0.32<$ all Pearson $\mathrm{r}$ correlations $<0.35$, all $p$ values $>0.13$ ). For pregabalin against placebo, there were some significant correlations $(-0.30<$ all Pearson $\mathrm{r}$ correlations $<0.56$, several $p$ values $<0.05$ ). These concerned the correlations between differences in baseline startle and the state anxiety score taken after the FPS procedure at $1 \mathrm{~h}$ $45 \mathrm{~min}$. This state anxiety measure correlated with baseline startle regardless of whether all trials were included in the baseline average (Pearson $r=0.56, p<$ 0.05 ) or just the trials in the neutral condition (Pearson $r=$ $0.53, p<0.05)$. This indicates a correlation between reduced state anxiety and baseline startle under the influence of pregabalin, which may be interpreted as indicative of an anxiolytic effect.

Skin conductance

There was a highly significant effect of context on the SCR response to the cues in the predictable versus neutral condition $(F(1,19 ; \mathrm{GG} \varepsilon=1)=25.1, p<0.001$, see Table 3$)$. In contrast to the drug effect on overall startle magnitude, there was no main effect of treatment on SCR responses ( $F$ $(3,57 ; \mathrm{GG} \varepsilon=0.95)=1.3$, NS $)$. Like startle, the effect of threat condition on SCR was not significantly affected by treatment (interaction between threat condition and treatment, $F(3,57 ; \mathrm{GG} \varepsilon=0.85)=1.1$, NS).

The onset of the unpredictable context evoked a SCR that was significantly larger than the SCR to the onset of the neutral context $(F(1,19 ; \mathrm{GG} \varepsilon=1)=39.0, p<0.001)$. As with the SCRs to the cues in the neutral and predictable conditions, there was no main effect of treatment on SCR to the onsets of the neutral and unpredictable contexts ( $F$ $(3,57 ; \mathrm{GG} \varepsilon=0.84)=0.5$, NS $)$. The effect of threat condition (unpredictable minus neutral context) was again not signif-

Table 3 SCR magnitudes

\begin{tabular}{|c|c|c|c|c|c|c|c|c|c|c|c|c|}
\hline & \multicolumn{6}{|c|}{ Cues in the neutral and predictable shock contexts } & \multicolumn{6}{|c|}{ Onsets of the neutral and unpredictable shock contexts } \\
\hline & \multicolumn{2}{|c|}{ Neutral cue } & \multicolumn{2}{|c|}{$\begin{array}{l}\text { Predictable } \\
\text { cue }\end{array}$} & \multicolumn{2}{|c|}{$\begin{array}{l}\text { Cue fear potentiation } \\
\text { (difference) }\end{array}$} & \multicolumn{2}{|c|}{$\begin{array}{l}\text { Neutral } \\
\text { context }\end{array}$} & \multicolumn{2}{|c|}{$\begin{array}{l}\text { Unpredictable shock } \\
\text { context }\end{array}$} & \multicolumn{2}{|c|}{$\begin{array}{l}\text { Context fear potentiation } \\
\text { (difference) }\end{array}$} \\
\hline & Mean & SEM & Mean & SEM & Mean & SEM & Mean & SEM & Mean & SEM & Mean & SEM \\
\hline Pla & 0.053 & 0.008 & 0.160 & 0.023 & 0.107 & 0.018 & 0.072 & 0.019 & 0.311 & 0.041 & 0.239 & 0.045 \\
\hline Alp & 0.072 & 0.014 & 0.155 & 0.034 & 0.083 & 0.028 & 0.101 & 0.028 & 0.234 & 0.052 & 0.133 & 0.052 \\
\hline Dip & 0.056 & 0.008 & 0.129 & 0.022 & 0.073 & 0.120 & 0.076 & 0.015 & 0.243 & 0.045 & 0.167 & 0.041 \\
\hline Prg & 0.073 & 0.011 & 0.176 & 0.031 & 0.103 & 0.024 & 0.071 & 0.020 & 0.298 & 0.045 & 0.227 & 0.039 \\
\hline
\end{tabular}

SEM standard error of the mean, Pla placebo, Alp alprazolam, Dip diphenhydramine, Prg pregabalin 
icantly affected by treatment (interaction between threat condition and treatment, $F(3,57 ; \mathrm{GG} \varepsilon=0.85)=1.8$, NS $)$.

\section{Discussion}

This study replicates earlier findings (Grillon et al. 2006) that alprazolam and diphenhydramine do not affect cuespecific FPS and extends these results in showing that pregabalin did also not affect FPS in reaction to a specific cue. In addition, we observed significant effects of alprazolam and diphenhydramine but not pregabalin on baseline startle. However, this study did not replicate the effect of alprazolam on context-potentiated startle found in the study by Grillon et al. (2006), thereby adding to the mixed results on pharmacological validation of the FPS reviewed in the "Introduction" section. These mixed results could be ground for disqualifying the FPS model as a screening tool for anxiolytic drugs. However, the FPS model offers the unique opportunity for direct translation from animals to humans (Grillon 2008). Even though pharmacological validation of the FPS model has not proven straightforward, there is no other human experimental model for anxiety available that has proven more successful (reviewed below). Therefore, finding the set of parameters that allows for robust (i.e., repeated) pharmacological validation of this model remains worthwhile.

What explanations may be possible for our unexpected null result? First, the lack of significant effects of alprazolam on context-specific FPS could be a false negative. Second, the positive results found by Grillon could have been due to chance. This is however unlikely given positive results in various other studies (Bitsios et al. 1999; Graham et al. 2005; Riba et al. 2001). Third, the different results could be explained by methodological dissimilarities between these studies in participants, experimental parameters, and measurements. Sensitivity of experimental models to varying parameters is not unprecedented (e.g., Davis et al. 1990), and these difficulties can be overcome if the important parameters are understood. In this case, it is important to understand which experimental variations make the difference between whether or not an anxiolytic effect of benzodiazepines in the human anxiety model based on fear-potentiated startle is observed.

The design of the present study replicated that of Grillon et al. (2006). However, comparison of the present study (parameters listed first) and the alprazolam study of Grillon et al. (2006, parameters listed second) revealed some minor differences between the methodologies used. The subject samples were comparable with similar age $(22.4 \pm 4.2$ versus $23.0 \pm 4.7)$ and levels of trait anxiety (31.8 \pm 7.3 versus $28.2 \pm 4.8$ ), but our study included relatively more men (ten men, ten women versus five men, 11 women). However, including Gender as a factor in the test for drug effects of context FPS did not change any of the conclusions (all interactions with and main effect of Gender $F<1$, NS). The parameters that were different in the stimulation protocol were the number of shock reinforcements ( 1 or 2 per condition versus 1$)$, the exact startle noise intensity and duration (105 dB(A), $50 \mathrm{~ms}$ versus $103 \mathrm{~dB}(\mathrm{~A}), 40 \mathrm{~ms})$, duration of time intervals between subsequent startle probes $(12-20 \mathrm{~s}$ versus $18-25 \mathrm{~s})$, duration of the contexts (90-100 s versus $150 \mathrm{~s}$ ), and timing of shocks in the unpredictable context (variable versus 7 or $10 \mathrm{~s}$ after cue offset).

Regardless of these differences, VAS rating scale data of subjective anxiety induced by the unpredictable versus the neutral context was comparable (4.0 versus 3.8$)$. Baseline startle magnitudes in absolute values were quite different, probably reflecting minor differences in preprocessing such as differences in smoothing factor (baseline startle under placebo in the neutral context $60.8 \pm 8.8 \mu \mathrm{V}$ versus $38.4 \pm$ $10.8 \mu \mathrm{V})$. Therefore, effects of cued fear and contextual anxiety on absolute startle levels differ. However, the size of cued FPS was remarkably similar when expressed in percentage increase (startle magnitude in predictable context without cue $69.2 \mu \mathrm{V}$ and with cue $85.5 \mu \mathrm{V}$, difference $16.3 \mu \mathrm{V}$ or $24 \%$ versus $47.2 \mu \mathrm{V}$ and $57.9 \mu \mathrm{V}$, difference $10.7 \mu \mathrm{V}$ or $23 \%$ ). The size of contextual FPS was larger in the study of Grillon et al. when expressed in percentage potentiation (startle magnitude in neutral context $60.8 \mu \mathrm{V}$ and unpredictable $78.8 \mu \mathrm{V}$, difference $18.0 \mu \mathrm{V}$ or $30 \%$ versus $38.4 \mu \mathrm{V}$ and $53.6 \mu \mathrm{V}$, difference $15.2 \mu \mathrm{V}$ or $40 \%$ ).

Any of the differences between the protocols could theoretically account for the difference in results. For example, duration of the conditions has been suggested as an important factor in the differentiation between cued and contextual responses (Walker et al. 2003), but it seems unlikely that the difference between 1.5 and 2.5 min would be crucial. A difference that may be of importance is the interval between subsequent startle probes, which was notably shorter in the present study compared to Grillon et al. (2006). Shortening these intervals does not abolish fear potentiation effects of cues (present results and Baas et al. 2002a, b) or contexts (present results and Böcker et al. 2004). Also, shortening these intervals does not affect all drug effects on startle negatively: In contrast to Grillon et al. (2006), who found no significant overall depressing effects of either benzodiazepine or diphenhydramine treatment, both substances markedly reduced baseline startle in the present study. However, even though changes in state anxiety may contribute importantly to an overall reducing effect of drug on startle suggestive of an anxiolytic effect (see Baas et al. 2002a), replication of this result was only marginally significant for alprazolam, and interpretation as anxiolysis is not applicable to diphenhydramine. Shorter 
intervals between startle stimuli lead to stronger habituation effects by allowing less recovery between trials, which might contribute to the sensitivity of baseline startle to the effects of drugs. Hypothetically, the difference between studies in sensitivity of baseline startle to drug treatments may be related to the differential sensitivity of the context potentiation effect to drug treatments observed. Of interest in this regard may be that while in Grillon et al. (2006) baseline startle was unaffected by drug, the context potentiation effect was relatively large. Summarizing, the theoretical possibility exists that shorter inter startle intervals influence sensitivity of startle modulation to benzodiazepines and possibly also context, which may be related to reduced sensitivity of context effects to benzodiazepines.

What may be promising is the finding that exclusion of two subjects who displayed a negative contextual anxiety effect under the placebo condition (as indicated by more startle reactivity during the neutral than during the unpredictable context) yielded a significant specific contrast with alprazolam. In the study by Grillon et al., there were several subjects who scored close to zero, but none who displayed a negative context effect, as the two observed in this study (not reported in Grillon et al. 2006; data were kindly provided by C. Grillon for further analyses). Nevertheless, high variability of startle results between sessions within subjects and between subjects is a more general problem that is not easily corrected by excluding subjects who fail to display the expected behavior under placebo. In future studies, a baseline measurement as part of the screening procedure to assess whether each subject displays the expected fear and anxiety reactions prior to being exposed to a drug treatment would be advisable, though this would not prevent variability in behavior within subjects across sessions.

An additional approach to reduce between-subjects variance caused by rather extreme differences in baseline startle between subjects is calculating $z$ or $T$ scores across all measurements per subject. The analyses on $T$ scores did not lead to a different conclusion in the present study. Nevertheless, the amount of variance between subjects and measurements within subjects observed in this study could be part of the explanation for the lack of drug effects. In our previous work, even though the drug manipulations did not affect FPS significantly, variation in baseline startle between drug conditions correlated significantly with differences in state anxiety and sedation as a result of drug administration (Baas et al. 2002a). For the alprazolam treatment in the present study, some of the drug effects on baseline startle may be explained by an anxiolytic effect, but the lack of strong correlations between baseline startle and state measures of anxiety and sedation may point to sources of variation that were not under control. The complexity of the design with respect to the simple threat/ safe manipulation in the previous study may have introduced additional variation. However, additional analyses on the data from Grillon et al. (2006) indicated that even though there were no correlations between baseline EMG and measures of anxiety and sedation for diphenhydramine or the low dose of alprazolam, these correlations were very significant in the high dose of alprazolam compared to placebo. As in our previous study, reductions in anxiety in Grillon et al. (2006) accounted for the largest part of variation in baseline startle (correlation $r=0.68, p<0.01$, Beta in regression model $=0.61, t=3.3, p<0.01)$, and sedation measures accounted for some variation (marginally significant, correlation $r=0.50, p=0.07$, Beta in regression model $=-0.41, t=-2.2, p=0.05)$. These results make it unlikely that complexity of the design in the present study with respect to our previous work (Baas et al. 2002a) can explain the lack of correlations between these measures in the present study.

These relatively weak associations between physiological and subjective data stand in contrast with the finding that alprazolam significantly reduced subjective ratings of contextual fearfulness. These results were rather similar in pattern to the effects on startle (significant reduction in overall startle/fearfulness with a marginally significant interaction with context), while usually, the FPS measures prove more sensitive than subjective ratings (Grillon et al. 2006; Grillon et al. 2007). This may indicate that variability in physiological data between subjects and sessions caused by factors that remain unexplained resulted in low power to detect effects of drugs on FPS in this study. If this explanation is correct, it appears that subtle differences in experimental conditions have a large impact on the sensitivity of the FPS paradigm to anxiolytic drugs. In this respect, it could be important that the study was performed in a clinical pharmacological rather than a psychological research institute-albeit under direct supervision of an experienced psychophysiologist under highly controlled circumstances. This research environment was a planned part of the more general validation of the FPS procedure to examine the applicability of this anxiety model for early drug development. Marked sensitivity to changing circumstances would disqualify a model for regulatory purposes. It would be important to identify and control the most important influential methodological factors before FPS can be considered a useful tool for anxiolytic drug development.

The lack of effect from pregabalin on any of the measures taken in the FPS experiment may possibly be related to the timing of the experiment. On pregabalin, subjects displayed a steady decline in state anxiety, which peaked around 4-6 h after dosing while theoretically, plasma concentrations peaked earlier (see "Methods" section). This effect had not yet reached significance at 
the time of the state anxiety measurements at $1 \mathrm{~h}$ and $1 \mathrm{~h}$ 45 min after dosing, between which the FPS test took place (data will be reported elsewhere). Though state anxiety under pregabalin was not yet significantly reduced with respect to baseline at the $1 \mathrm{~h} 45$ min measurement, decrease of state anxiety under pregabalin compared to placebo showed a significant correlation with decreases in baseline startle from placebo to pregabalin. Therefore, some anxiolytic features were observed, but the peak of pregabalin's effect on state anxiety occurred well after the FPS measurements took place. A negative finding in a drug study in which the efficacy is measured at only one time point after drug administration leaves open the possibility that the effects were not measured at the peak of the drug's effect in the central nervous system. This is especially problematic in the study of drugs of which the pharmacokinetics are still largely unknown, as is the case in early screening of new drugs. The FPS model could gain usability if it could be tested at several moments after drug administration. This would require frequent repetition of the FPS model, including several measurements per test session after drug administration on several different study occasions to allow full cross-over comparative designs of different doses or treatments. We have recently developed such a design (Klumpers et al. 2009).

Finally, the present results again showed that diphenhydramine can be used as a sedative control substance for FPS validation studies with benzodiazepines. Nevertheless, the problem remains that effects of sedation and anxiolysis are hard to disentangle. Ideally, new anxiolytic drugs should not suffer from sedative side effects. Future validation studies would benefit from inclusion of non-sedative anxiolytic substances. To better understand GABA-ergic effects in human FPS models, examination of novel subtype-selective GABA-agonists would be useful. These experimental drugs selectively activate the $\alpha 2$-subunit of the $\mathrm{GABA}_{\mathrm{A}}$ receptor, which is thought to be responsible for the anxiolytic effects of benzodiazepines (Low et al. 2000), without having affinity for the $\alpha 1$-subunit considered to be responsible for the sedative effects (Rudolph et al. 1999). Recent publications suggest that the availability of such substances may not be too far ahead in the future (Atack et al. 2006; de Haas et al. 2007; de Haas et al. 2008).

How do the mixed results in validating the fearpotentiated startle paradigm pharmacologically compare to validation of other human experimental models for anxiety? Besides the FPS model, three other human experimental models for anxiety have been subjected to repeated attempts at pharmacological validation. Conditioned skin conductance responses are affected by benzodiazepines, but mostly because overall responses are subject to dampening by drug treatments (Hellewell et al. 1999, see also Graeff et al. 2003). Significantly reduced conditioned responses may therefore be caused by floor effects. Even though baseline startle is also affected by drug manipulations, the reflex remains strong enough to avoid floor effects (Davis et al. 1993). A more recent study of conditioned fear using fearpotentiated startle as a read-out measure did not find an effect of diazepam on the expression of conditioned fear (Scaife et al. 2005).

Models of social stress such as public speaking have also been studied with mixed results. Benzodiazepines reduce overall anxiety but do not affect the increase in anxiety as a result of the challenge, and effects of serotonergic manipulations are varied (see Graeff et al. 2003). The Trier social stress test showed significant effects of alprazolam in blunting endocrine stress reactions, without effects on psychological measures (Fries et al. 2006). A drawback with tests as these is that subjects' response habituation prevents their use in cross-over designs (Klumpers et al. 2009).

A model proposed by Bailey et al. (2005) based on anxiety induction by inhalation of $\mathrm{CO}_{2}$ was subjected to pharmacological validation in several recent studies. Inhalation of low $\mathrm{CO}_{2}$ concentrations $(7.5 \%)$ has been proposed as a model for generalized anxiety (Bailey et al. 2005). Benzodiazepines lorazepam (2 mg) and alprazolam (1 mg) reduced subjective but not cardiovascular effects while paroxetine had little effect (Bailey et al. 2007, 2009), and propanolol, hydroxyzine, and flupentixol had no subjective effects even though propanolol decreased heart rate (Papadopoulos et al. 2008).

In conclusion, the present study could not confirm the dissociation found in effects of alprazolam on contextual fear but not cued fear. The one obvious conclusion to be drawn from these results is that the FPS model, as is the case with pharmacological validation of experimental models for anxiety in general, is by no means easily implemented and validated. Recommendations for future FPS studies are inclusion of a baseline measurement as part of the screening to exclude subjects who do not display FPS, which is after all the measure of interest. The model would benefit from further development in order to reduce variance in the startle response and FPS. Also, models including multiple measurements spread out over time would improve chances of finding effects of a drug manipulation, especially with drugs of which the pharmacokinetic profile is still unknown. Finally, possible effects of the length of the interval between startle stimuli deserve further investigation.

Acknowledgment The study was supported by a restricted grant from Hoffmann-La Roche, Basel, Switzerland.

Open Access This article is distributed under the terms of the Creative Commons Attribution Noncommercial License which permits any noncommercial use, distribution, and reproduction in any medium, provided the original author(s) and source are credited. 


\section{References}

Atack JR, Wafford KA, Tye SJ, Cook SM, Sohal B, Pike A, Sur C, Melillo D, Bristow L, Bromidge F, Ragan I, Kerby J, Street L, Carling R, Castro JL, Whiting P, Dawson GR, McKernan RM (2006) TPA023 [7-(1, 1-dimethylethyl)-6-(2-ethyl-2H-1, 2, 4triazol-3-ylmethoxy)-3-(2-fluor ophenyl)-1, 2, 4-triazolo[4, 3-b] pyridazine], an agonist selective for alpha2- and alpha3containing GABAA receptors, is a nonsedating anxiolytic in rodents and primates. J Pharmacol Exp Ther 316:410-422

Baas JM, Grillon C, Böcker KB, Brack AA, Morgan CA 3rd, Kenemans JL, Verbaten MN (2002a) Benzodiazepines have no effect on fear-potentiated startle in humans. Psychopharmacology (Berl) 161:233-247

Baas JM, Kenemans JL, Böcker KB, Verbaten MN (2002b) Threatinduced cortical processing and startle potentiation. Neuroreport 13:133-137

Bailey JE, Argyropoulos SV, Kendrick AH, Nutt DJ (2005) Behavioral and cardiovascular effects of $7.5 \% \mathrm{CO} 2$ in human volunteers. Depress Anxiety 21:18-25

Bailey JE, Kendrick A, Diaper A, Potokar JP, Nutt DJ (2007) A validation of the $7.5 \% \mathrm{CO} 2$ model of GAD using paroxetine and lorazepam in healthy volunteers. J Psychopharmacol 21:42-49

Bailey J, Papadopoulos A, Seddon K, Nutt DJ (2009) A comparison of the effects of a subtype selective and non-selective benzodiazepine receptor agonist in two $\mathrm{CO} 2$ models of experimental human anxiety. J Psychopharmacol 23:117-122

Bitsios P, Philpott A, Langley RW, Bradshaw CM, Szabadi E (1999) Comparison of the effects of diazepam on the fear-potentiated startle reflex and the fear-inhibited light reflex in man. J Psychopharmacol 13:226-234

Blumenthal TD, Cuthbert BN, Filion DL, Hackley S, Lipp OV, van Boxtel A (2005) Committee report: guidelines for human startle eyeblink electromyographic studies. Psychophysiology 42:1-15

Blyden GT, Greenblatt DJ, Scavone JM, Shader RI (1986) Pharmacokinetics of diphenhydramine and a demethylated metabolite following intravenous and oral administration. J Clin Pharmacol 26:529-533

Böcker KB, Baas JM, Kenemans JL, Verbaten MN (2004) Differences in startle modulation during instructed threat and selective attention. Biol Psychol 67:343-358

Bond A, Lader M (1974) The use of analogue scales in rating subjective feelings. Br J Med Psychol 47:211-218

Davis M, Falls WA, Campeau S, Kim M (1993) Fear-potentiated startle: a neural and pharmacological analysis. Behav Brain Res 58:175-198

Davis M, Mansbach RS, Swerdlow NR, Campeau S, Braff DL, Geyer MA (1990) Apomorphine disrupts the inhibition of acoustic startle induced by weak prepulses in rats. Psychopharmacology (Berl) 102:1-4

de Haas SL, de Visser SJ, van der Post JP, de Smet M, Schoemaker RC, Rijnbeek B, Cohen AF, Vega JM, Agrawal NG, Goel TV, Simpson RC, Pearson LK, Li S, Hesney M, Murphy MG, van Gerven JM (2007) Pharmacodynamic and pharmacokinetic effects of TPA023, a $\operatorname{GABA}(\mathrm{A})$ alpha(2, 3) subtype-selective agonist, compared to lorazepam and placebo in healthy volunteers. J Psychopharmacol 21:374-383

de Haas SL, de Visser SJ, van der Post JP, Schoemaker RC, van Dyck K, Murphy MG, de Smet M, Vessey LK, Ramakrishnan R, Xue L, Cohen AF, van Gerven JM (2008) Pharmacodynamic and pharmacokinetic effects of MK-0343, a GABA(A) alpha2, 3 subtype selective agonist, compared to lorazepam and placebo in healthy male volunteers. J Psychopharmacol 22:24-32

Fries E, Hellhammer DH, Hellhammer J (2006) Attenuation of the hypothalamic-pituitary-adrenal axis responsivity to the Trier
Social Stress Test by the benzodiazepine alprazolam. Psychoneuroendocrinology 31:1278-1288

Garzone PD, Kroboth PD (1989) Pharmacokinetics of the newer benzodiazepines. Clin Pharmacokinet 16:337-364

Graeff FG, Parente A, Del-Ben CM, Guimarães FS (2003) Pharmacology of human experimental anxiety. Braz J Med Biol Res $36: 421-432$

Graham SJ, Scaife JC, Langley RW, Bradshaw CM, Szabadi E, Xi L, Crumley T, Calder N, Gottesdiener K, Wagner JA (2005) Effects of lorazepam on fear-potentiated startle responses in man. J Psychopharmacol 19:249-258

Grillon C (2002) Startle reactivity and anxiety disorders: aversive conditioning, context, and neurobiology. Biol Psychiatry 52:958975

Grillon C (2008) Models and mechanisms of anxiety: evidence from startle studies. Psychopharmacology (Berl) 199:421-437

Grillon C, Baas JM (2002) Comments on the use of the startle reflex in psychopharmacological challenges: impact of baseline startle on measurement of fear-potentiated startle. Psychopharmacology (Berl) 164:236-238

Grillon C, Baas JM (2003) A review of the modulation of the startle reflex by affective states and its application in psychiatry. Clin Neurophysiol 114:1557-1579

Grillon C, Ameli R, Woods SW, Merikangas K, Davis M (1991) Fearpotentiated startle in humans: effects of anticipatory anxiety on the acoustic blink reflex. Psychophysiology 28:588-595

Grillon C, Ameli R, Merikangas K, Woods SW, Davis M (1993) Measuring the time course of anticipatory anxiety using the fearpotentiated startle reflex. Psychophysiology 30:340-346

Grillon C, Cordova J, Levine LR, Morgan CA, 3rd (2003) Anxiolytic effects of a novel group II metabotropic glutamate receptor agonist (LY354740) in the fear-potentiated startle paradigm in humans. Psychopharmacology (Berl) 168:446-454

Grillon C, Baas JM, Lissek S, Smith K, Milstein J (2004) Anxious responses to predictable and unpredictable aversive events. Behav Neurosci 118:916-924

Grillon C, Baas JM, Pine DS, Lissek S, Lawley M, Ellis V, Levine J (2006) The benzodiazepine alprazolam dissociates contextual fear from cued fear in humans as assessed by fear-potentiated startle. Biol Psychiatry 60:760-766

Grillon C, Levenson J, Pine DS (2007) A single dose of the selective serotonin reuptake inhibitor citalopram exacerbates anxiety in humans: a fear-potentiated startle study. Neuropsychopharmacology 32:225-231

Hellewell JS, Guimaraes FS, Wang M, Deakin JF (1999) Comparison of buspirone with diazepam and fluvoxamine on aversive classical conditioning in humans. J Psychopharmacol 13:122127

Hermans EJ, Putman P, Baas JM, Koppeschaar HP, van Honk J (2006) A single administration of testosterone reduces fear-potentiated startle in humans. Biol Psychiatry 59:872-874

Klumpers F, van Gerven JM, Prinssen EPM, Niklson I, Roesch F, Riedel WJ, Kenemans JL, Baas JMP (2009) Method development studies for repeatedly measuring anxiolytic drug effects in healthy humans. J Psychopharmacol doi:10.1177/0269881109103115

Low K, Crestani F, Keist R, Benke D, Brunig I, Benson JA, Fritschy JM, Rulicke T, Bluethmann H, Mohler H, Rudolph U (2000) Molecular and neuronal substrate for the selective attenuation of anxiety. Science 290:131-134

Mol N, Baas JM, Grillon C, van Ooijen L, Kenemans JL (2007) Startle potentiation in rapidly alternating conditions of high and low predictability of threat. Biol Psychol 76:43-51

Papadopoulos A, Rich A, Nutt DJ, Bailey JE (2008) The effects of single dose anxiolytic medication on the $\mathrm{CO} 2$ models of anxiety: differentiation of subjective and objective measures. J Psychopharmacol doi:10.1177/0269881108097716 
Paton DM, Webster DR (1985) Clinical pharmacokinetics of H1receptor antagonists (the antihistamines). Clin Pharmacokinet 10:477-497

Patrick CJ, Berthot BD, Moore JD (1996) Diazepam blocks fearpotentiated startle in humans. J Abnorm Psychol 105:89-96

Riba J, Rodriguez-Fornells A, Urbano G, Antonijoan R, Barbanoj MJ (1999) Fear potentiated startle of the acoustic startle response is preserved after lorazepam administration to human subjects. Psychophysiology 36:S94

Riba J, Rodriguez-Fornells A, Urbano G, Morte A, Antonijoan R, Barbanoj MJ (2001) Differential effects of alprazolam on the baseline and fear-potentiated startle reflex in humans: a dose-response study. Psychopharmacology (Berl) 157:358367

Rudolph U, Crestani F, Benke D, Brunig I, Benson JA, Fritschy JM, Martin JR, Bluethmann H, Mohler H (1999) Benzodiazepine actions mediated by specific gamma-aminobutyric acid(A) receptor subtypes. Nature 401:796-800

Scaife JC, Langley RW, Bradshaw CM, Szabadi E (2005) Diazepam suppresses the acquisition but not the expression of 'fearpotentiation' of the acoustic startle response in man. J Psychopharmacol 19:347-356

Spielberger CD (1972) Manual for the State-Trait Anxiety Inventory. Consulting Psychologist Press, Washington

Stahl SM (2004) Mechanism of action of alpha2delta ligands: voltage sensitive calcium channel (VSCC) modulators. J Clin Psychiatry 65:1033-1034

Walker DL, Toufexis DJ, Davis M (2003) Role of the bed nucleus of the stria terminalis versus the amygdala in fear, stress, and anxiety. Eur J Pharmacol 463:199-216

Zareba G (2005) Pregabalin: a new agent for the treatment of neuropathic pain. Drugs Today 41:509-516 